MATEC Web of Conferences 8, 01003 (2013)

DOI: $10.1051 /$ matecconf $/ 20130801003$

(C) Owned by the authors, published by EDP Sciences, 2013

\title{
Dynamic optics for ultrafast laser processing
}

\author{
Patrick Salter $^{1}$ and Martin Booth ${ }^{1,2}$ \\ ${ }^{1}$ Department of Engineering Science, University of Oxford, Parks Road, Oxford OX1 3PJ, United Kingdom \\ ${ }^{2}$ Centre for Neural Circuits and Behaviour, Tinsley Building, Mansfield Road, Oxford, OX1 3SR, United Kingdom \\ martin.booth@eng.ox.ac.uk
}

\begin{abstract}
We present a range of dynamic optical methods to control focal fields for material processing using ultrafast lasers. Adaptive aberration correction maintains focal quality when focusing deep into materials. Dynamic parallelisation methods permit independent control of hundreds of fabrication spots. New adaptive methods for control of pulse front tilt are also presented.
\end{abstract}

OCIS codes: 010.1080 Active or adaptive optics, 140.3390 Laser materials processing

\section{Introduction}

Laser material processing with short pulsed laser beams has been widely used for the fabrication of devices such as artificial bandgap materials [1], microfluidic devices [2], metal nanostructures [3] and photonic devices based upon embedded waveguides [4]. Structures are created by focusing the laser into the material where multi-photon absorption and/or avalanche effects cause permanent material changes in the focal region. We present new methods that use dynamic optical elements - deformable mirrors and liquid crystal spatial light modulators - to bring extra degrees of control to the laser fabrication system. The methods include aberration correction, parallelisation and control of pulse front tilt. Examples are presented of 3D waveguides in silica, millimetre scale photonic crystals, and fine structures deep inside diamond.

\section{Aberration correction}

The fidelity of fabrication in laser processing depends strongly on the focal spot quality, which in many cases is impaired by aberrations. A common source of aberration is a mismatch between the refractive indices of the processed material and the objective immersion medium. For example, photonic devices are often fabricated in glasses that are not index matched with the focusing objective. Aberrations lead to a deviation from the desired focal energy distribution, in particular an elongation along the optic axis, and a reduction in its intensity. As a consequence, the accuracy of fabrication is compromised. As one focuses deeper into the substrate, the aberrations increase in magnitude. We have previously shown that adaptive optics can compensate for this spherical aberration, thus restoring diffraction limited fabrication at any point in the substrate [5]. Here, we develop the adaptive optical system for the parallel generation of 3D arrays of fabrication spots, which are individually corrected for aberration and provide demonstrations in a range of fabrication substrates including various glasses, diamond and lithium niobate.

\section{Adaptive parallel fabrication}

In many ultrafast laser fabrication systems, sufficient laser power is available in principle to support many fabrication foci in parallel, thus enabling reduced fabrication times. This can be done through the use of a microlens array [6], space-time focusing [7] or holography [8]. The approach used here is to display phase-only holograms on a liquid crystal spatial light modulator. This allows the generation of 3D aperiodic arrays of fabrication spots that reduces production times for complex structures. Furthermore, it provides the ability to simultaneously correct for any optical aberrations in the system. This is implemented through incorporation of phase correction into the hologram design, where the degree of correction is adjusted for each focusing depth in the array. Rapid fabrication of large complex structures becomes possible (Fig. 1).

\section{Dynamic optics for waveguide fabrication}

More complex aberrations are experienced when focusing near the edge of the chip, as a proportion of the focused light is refracted by the side surface. This results in a focal splitting phenomenon that leads to a highly distorted waveguide structure near the side. In practice, writing has been terminated before the edge and the chip has been cut and polished up to the waveguide. We implement an adaptive optics system that corrects light passing through both the top and side surfaces [9]. This permits writing up to the side face of the waveguide chip, removing the need for cutting and polishing, thus reducing the time required to produce a waveguide circuit. Further increase in fabrication speed can be achieved by parallelisation of the writing process. We describe adaptive methods where multiple controllable foci are used to create single waveguides with tunable cross-sections. 


\section{Adaptive control of pulse front tilt}

The "quill effect" describes a curious directional phenomenon frequently encountered during ultrafast fabrication of subsurface continuous structures. Even in a homogeneous and isotropic material, such as fused silica, the fabrication regime experienced can be dependent on the direction of motion relative to the optic axis. The directionality has been attributed to a tilt of the pulse front relative to the incident wavefront, leading to a spatiotemporal asymmetry in the focal plane. Here we use a liquid crystal spatial light modulator (SLM) to demonstrate and control the quill effect when using a femtosecond laser for bulk fabrication of fused silica [10]. A pulse front tilt can be introduced to the fabrication beam by applying a blazed grating to the SLM, and tuned by varying the grating periodicity. As a result, the fabricated features generated when moving the substrate in opposite directions relative to the tilt are different. Reversing the blazed grating switches the effect, confirming the fabrication dependence on the pulse front tilt. However, it is additionally shown that inhomogeneous pupil illumination can cause similar directionality in the fabrication, through a simple spatial asymmetry in the focal intensity distribution. We show that the pupil illumination profile can likewise be controlled adaptively by the SLM and that directional effects can be dynamically introduced during fabrication. Further possibilities are considered for higher order control of ultrashort pulses using combinations of adaptive optical elements.

(a)

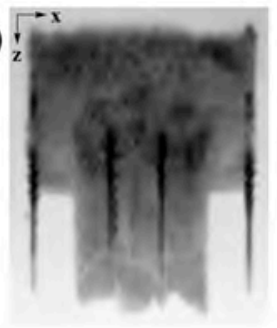

(c)

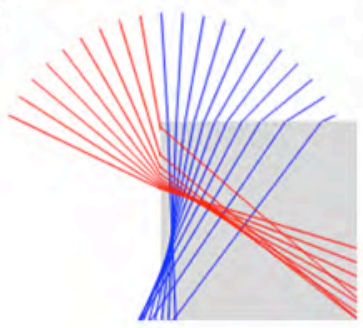

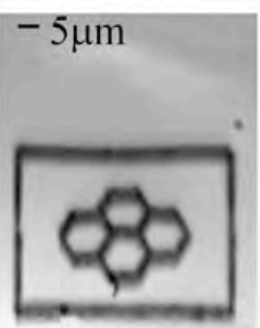

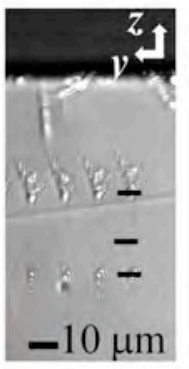

(b)

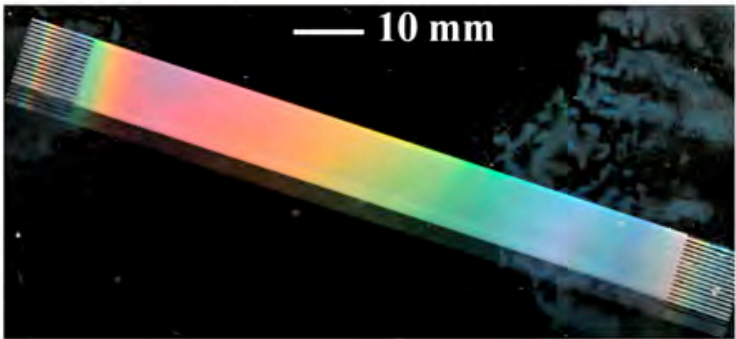

(d)

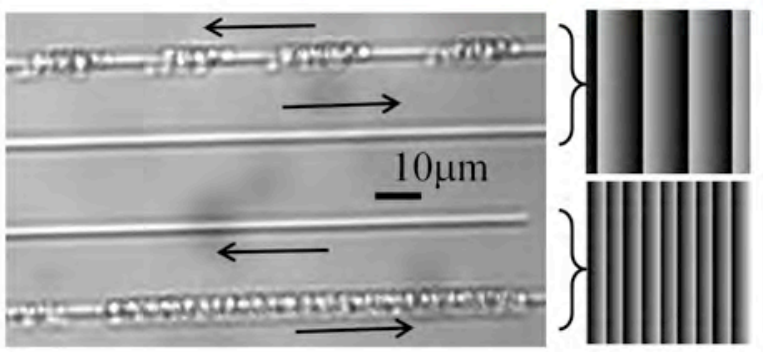

Fig. 1(a) Aberration correction enables controlled laser writing of fine features deep with diamond. (b) Parallel processing enables the rapid fabrication of large volume optics. (c) Focal splitting due to refraction at the substrate edge can be corrected, benefitting waveguide writing and (d) a SLM may be used to control pulse front tilt and directional effects in fabrication.

\section{References}

[1] S. Wong, M. Deubel, F. Perez-Willard, S. John, G. A. Ozin, M. Wegener, and G. von Freymann,, "Direct Laser Writing of ThreeDimensional Photonic Crystals with a Complete Photonic Bandgap in Chalcogenide Glasses," Adv. Mater. 18, 265-269 (2006).

[2] R. Osellame, V. Maselli, R. Martinez Vazquez, R. Ramponi and G. Cerullo, "Integration of optical waveguides and microfluidic channels both fabricated by femtosecond laser irradiation," Appl. Phys. Lett. 90, 231118 (2007)

[3] M. S. Rill et al., "Photonic metamaterials by direct laser writing and silver chemical vapour deposition," Nature Materials 7, 543-546 (2009).

[4] G. Della Valle, R. Osellame and P. Laporta, “Micromachining of photonic devices by femtosecond laser pulses,” J. Opt. A 11, 013001 (2009).

[5] A. Jesacher et al., “Adaptive optics for direct laser writing with plasma emission aberration sensing,” Opt. Exp., 18, 656 (2010)

[6] J. Kato et al., "Multiple-spot parallel processing for laser micronanofabrication” App. Phys. Lett., 86, 044102 (2005)

[7] D. Kim and P.T.C. So, "High-throughput three-dimensional lithographic microfabrication" Opt. Lett. 35, 1602 (2010)

[8] A. Jesacher and M.J. Booth, "Parallel direct laser writing in three dimensions with spatially dependent aberration correction" Opt. Exp. 18, $21090(2010)$

[9] P.S. Salter and M.J. Booth, "Focussing over the edge: adaptive subsurface laser fabrication up to the sample face”, Opt. Express 20, 1997819989(2012)

[10] P.S. Salter and M.J. Booth, "Dynamic control of directional asymmetry observed in ultrafast laser direct writing," Appl. Phy. Lett. 101, 141109, (2012). 